\title{
Single Burr Hole Craniostomy in surgical treatment of chronic subdural hematoma (Outcome and Complications)
}

\author{
Ali T. AbdulWahid* MBChB, FIBMS
}

\begin{abstract}
:
Background: There is controversy among neurosurgeons whether double Burr Hole craniostomy is better than single Burr Hole, craniostomy in the management of chronic subdural hematoma in terms of lower recurrence rate and complications.

Objective: To assess the outcome and complications including revision rate using Single Burr Hole Craniostomy in the treatment of chronic subdural hematoma (CSDH).

Patients and Methods: This is a retrospective study of 42 cases of chronic subdural hematoma who underwent Single Burr Hole Craniostomy(SBHC) from jan.2012 to jan.2014, preoperative,intraoperative and postoperative data were collected including neurological status ,Brain CT-scan findings, postoperative complications ,recurrent rate and neurological deficit.

Fac Med Baghdad 2015; Vol.57, No.3 Received:March,2015 Accepted: April,2015

Results: Forty two patients (36 male and 6 female, with age ranged between $60 y .-82 y$.$) were treated by$ Single Burr Hole Craniostomy for chronic subdural hematoma. There were two recurrences, one stroke, and one death after primary surgery. The two recurrent cases were treated by drainage through same Burr Hole and both they did well (recovered uneventfully).

Conclusions: The results of this study suggest that Single Burr Hole Craniostomy is safe, simple and effective method for surgical management of CSDH with lower recurrence rate and lower morbidity and mortality.

Key Words: Chronic subdural hematoma (CSDH), Single Burr Hole Craniostomy (SBHC), complications.
\end{abstract}

\section{Introduction:}

$\mathrm{CSDH}$ is one of the most common neurosurgical problems that can be usually treated by simple and effective surgical procedure. $(1,2)$, however the perfect surgical method continues to attract debate (3), CSDH affects the elderly patients more than 65years of age who expected to double worldwide between 2000and 2030, so a corresponding rise in the incidence of CSDH.(4), CSDH has a male to female ratio of $3: 1$, usually presented with abnormal gait ,mental abnormality , headache, limbs weakness and acute decrease in the level of consciousness in $10 \%$ to $20 \%$, with history of mild head injury in $50 \%$ to $70 \%$.(5), Surgical drainage of CSDH can be achieved via craniotomy, Burr Hole craniostomy (5-30mm size) or twist drill craniostomy(less than $5 \mathrm{~mm}$ size).

Local or general anesthesia can be used and can be performed in theater or at bed side with numerous variations of each technique have become developed and are practiced. $(2,6)$, Burr Hole Craniostomy is the most effective option to treat uncomplicated CSDH because it balances a lower recurrent rate against morbidity and mortality better than craniotomy and twist drill craniostomy. $(7, .8)$, the use of drain with $\mathrm{BHC}$ is recommended and several studies demonstrated benefit from intraoperative irrigation and postoperative closed drainage associated with lower recurrence rate (9). MostsurgeonsuseddoubleBurrHoleCraniostomytoallowbetter

*Dept. of surgery, Medical College-Baghdad University.

E-mail:azdh-1978@yahoo.com washout of the subdural cavity and have found higher recurrence rate of single rather than double Burr Hole Craniostomy $(10,11)$, Data suggest that there is a role for postoperative bed rest for three days and lower recurrence rate in supine position group $2.3 \%$ and in the sitting position group $19 \%(12,13)$, Steroid and Angiotensin converting enzyme(ACE) inhibiter may be play a role in the management of CSDH.(14, 15) Craniotomy is used in patients with recollected subdural hematoma, solid, septated hematoma or when the brain fails to obliterate the subdural space.(2 16) Twist Burr Hole craniostomy was similar to $\mathrm{BHC}$, regarding mortality and morbidity, with higher recurrence rate than $\mathrm{BHC}$, but it can be done at bed side $(1,17,18)$.

\section{Patients and methods:}

Forty two patients with symptomatic chronic subdural hematoma were studied retrospectively who underwent surgical drainage from Jan. 2012 to Jan. 2014 at (nursing home hospital and surgical specialty hospital)-medical city and al -alwiya private hospital.

All patients were examined clinically and neurologically and brain CT-scan was obtained preoperatively.

Laboratory test include coagulation test were done for all patients,

Only single Burr Hole drainage was done for all cases of CSDH in this series except one patient who had bilateral chronic subdural hematoma who was treated by single Burr 
Hole drainage on each side.

Single Burr Hole drainage was done on the thickest part of $\mathrm{CSDH}$ as showed by brain CT-scan without intraoperative irrigation or postoperative drainage.

Drainage of CSDH intraoperativly was done by special maneuver, tilting operative table up and down, left and right until almost complete subdural hematoma evacuated.

Almost all patients had local anesthesia with conscious sedation but only two patients received general anesthesia because they were agitated and not cooperative. Steroid and antibiotics were given preoperatively and postoperatively for three days with bed rest in supine position and blood thinning medications were stopped. Patient was discharged home next day from hospital and seen one week for follow-up and removal of stitches. All patients were seen within one to two months, and most patients did well regarding their mental state, limb weakness and gait. Two patients presented with recurrent collection after primary surgery and drainage was done through the same single Burr Hole craninostomy.

One patient had stroke with persistent dense hemiplegic limbs who had uncontrolled hypertension, diabetes mellitus and ischemic heart diseases.

One patient died due to unknown causes one month after primary surgery.

Results:

Table 1 : presentations of patients with CSDH

\begin{tabular}{ccc}
\hline Presentation & CSDH patients & Incidence \\
\hline Gait disturbance & 15 & $35.8 \%$ \\
\hline Limbs weakness & 11 & $26.2 \%$ \\
\hline Mental deterioration & 10 & $23.8 \%$ \\
\hline Speech impairment & 6 & $14.2 \%$ \\
\hline
\end{tabular}

Fifteen patients $(35.7 \%)$ presented with gait disturbance, limbs weakness in 11 patients $(26.2 \%)$, mental deterioration in 10 patients $(23.8 \%)$, and speech impairment in six patients $(14.2 \%)$.

Table 2 : History of head trauma in patients with CSDH

\begin{tabular}{|c|c|c|}
\hline & CSDH patients & Incidence \\
\hline Head trauma & 32 & $76.2 \%$ \\
\hline No head trauma & 10 & $23.8 \%$ \\
\hline
\end{tabular}

History of mild trauma was elicited in 32 patients $(76 \%)$ and 10 patients no history of trauma, one patient had diabetes mellitus, one had epilepsy and two cases were alcoholic and heavy smoker.

Antihypertensive, antiplatelets and anticoagulants drugs were received by 20 patients $47.6 \%$ for long time.

Brain CT-scan showed low density crescent shape CSDH with midline shift, mass effect, with evidence of brain atrophy in almost all patients before surgery.
After surgery brain CT-scan showing decrease size of chronic subdural hematoma without midline shift or mass effect, but there was residual small collection which is not significant.

Table 3 : Postoperative sequelae after Single Burr Hole Craniostomy of CSDH

\begin{tabular}{|c|c|c|}
\hline Postoperative Sequelae & Patients CSDH & Percentage \\
\hline Uneventful & 38 & $90.6 \%$ \\
\hline Recurrent of CSDH & 2 & $4.8 \%$ \\
\hline Infarction & 1 & $2.3 \%$ \\
\hline Death & 1 & $2.3 \%$ \\
\hline
\end{tabular}

Two patients had recurrence of CSDH after few days of surgery and evacuation of hematoma was done for both patients through same Burr Hole craniostomy. One patient had cerebral infarction postoperatively as shown by brain CT-scan with resolve $\mathrm{CSDH}$, and One patient died one month after primary surgery due to unknown cause.

\section{Discussion:}

Burr Hole Crainostomy has been identified by Lega et.al (6) as the most efficient choice to treat symptomatic chronic subdural hematomas agree with this study. In this series, similar to most published series, the age distribution ranged between 60 years -82 years. (7) There was strong male preponderance $(85.8 \%)$ and $(14.2 \%)$ female in our group which is higher than recent published papers where male to female ratio $3: 1 .(7,8)$ History of trauma was elicited in 32 patients $(80 \%)$ which higher than other series (50\%-70\%). (5) The most cases presented in our study of CSDH was gait disturbance in 15 patients, limb weakness in 13, mental changes in 10 and stroke like in 6 patients which agree with Mori K,Maeda M 500 consecutive cases.(5) Most neurosurgeons use two Burr Hole for CSDH to allow better washout of subdural cavity and have found higher recurrence rate if single than double Burr Hole was used as shown by Sirajeddin Belkhair et.al. (10) contrast, the series by Han et.al (11), the recurrent rate was $2 \%$ if single Burr Hole and $7 \%$ if two Burr Holes were used which agree with our study, where the recurrence rate was $(4.8 \%)$ two patients out of $42 \mathrm{CSDH}$ treated by single Burr Hole, then two recurrent cases were treated by drainage through the same single Burr Hole and they recovered fully next day. Most neurosurgeons in UK don $>\mathrm{t}$ use postoperative drainage which is similar to policy (13), where no drain was applied postoperative. Several groups have demonstrated benefit from intra-operative irrigations, but Kuroki et.al found six times higher recurrence rate (11\%) in cases with irrigation than the cases without irrigation $(1.8 \%)$ $(19,20)$. One patient died in our study $(2 \%)$ one month after surgery with unknown causes, and one patient ( $2 \%)$ developed cerebral infarction with worsening of her neurological deficit postoperative as confirmed by brain CT-scan. Apart from those two patients 40 patients with CSDH treated by single Burr Hole 
craniostomy was recovery remarkably regarding mental state, limb weakness and gait disturbance. 20 patients out of 42 were receiving antiplatelets and anticoagulants which were stopped for one month after surgery. Abouzari et.al $(21,22)$ found the recurrent rate in the supine group $(2.3 \%)$ and $(19 \%)$ in sitting group which agree with our result who were lying supine in bed for 24 hours postoperatively with $4.8 \%$ recurrence rate of CSDH.

\section{Conclusions:}

Single Burr Hole craniostomy is safe, single, and effective, in surgical treatment of chronic subdural hematoma. It is associated with lower recurrence rate, lower morbidity and mortality.Neither intraopreative irrigations nor postoperative closed drainage were applied, and recommended in surgery of chronic subdural hematoma.

\section{References:}

1. Weigel R, Schmirdek P, Krauss JK. Outcome of contemporary surgery for chronic hematoma: evidence based review \{comment\}.J Neurol Neurosurg Psychiatr. 2003; 74(7):937-943.

2. Markwaalder TM. Chronic subdural hematomas:a review .J Neurosurg. 1981; 45:637-645.

3. Lee JK, Choi JH, Kim CH, Lee Hk, Moon JG Chronic subdural hematomas: a comparative study of the three types of operative procedures. J Korean Neurosurg Soc. 2009; 46(3):210-214.

4. Kinsella K, Velkoff VA. An Aging World: 2001. Washington, DC; US Government Printing Office; 2001. US Census Bureau Series P95/01-1, 9.

5. Mori K, Maeda M. Surgical treatment of chronic subdural hematoma in 500 consecutive cases: clinical characteristics, surgical outcome, complications and recurrence rate. Neurol Med Chir (Tokyo).2001; 41(8):371-381.

6. Lega BC, Danish SF, Malhotra NR, Sonnad SS, Stein SC. Choosing the best operation for chronic subdural hematoma: a decision analysis \{published online ahead of print October 30, 2009\}. J Neurosurg. DOI:10.3171/2009.9.JNSO8825.

7. Sambasivan M. An overview of chronic subdural hematoma: experience with 2300 cases. Surg Neurol. 1997; 47(5):418-422.

8. Lind CR, Lind CJ, Mee EW. Reduction in the number of repeated operations for the treatment of sub acute and chronic subdural hematomas by placement of subdural drains. $J$ Nerosurg. 2003; 99(1):44-46.

9. MondorfY, Abu-Owaimer M, Gaab MR, Oertel JM. Chronic subdural hematoma-Craniotomy versus burr hole trepanation. Br J Neurosurg. 2009; 23(6):612-616.

10. Sirajeddin Belkhair, Gwynedd Pickett. (2013) one versus double burr holes for treating chronic subdural hematoma meta- analysis. The Canadian journal of neurological science40, 56-60. Online publication date: 1-jan-2013 \{IVSL, high wire?.

11. Han HJ, Park CW, Kim EY, Yoo CJ, Kim YB, Kim WK. One vs. two burr hole craniostomy in surgical treatment of chronic subdural hematoma. J Korean Neurosurg Soc. 2009:46(2):8792.

12. Cenic A, Bhandari M, Reddy K. Management of chronic subdural hematoma: a national survey and literature review. Can J Neurol Sci. 2005; 32(4):501-506.

13. Santarius T, Lawton R, Kirkpatrick PJ, Hutchinson PJ. The management of primary chronic subdural hematoma: a questionnaire survey of practice in United Kingdom and the republic of Ireland. Br J Neurosurg. 2008; 22(4): 529-534.

14. Goksu E, Akyuz M, Ucar T, Kazan S. Spontaneous resolution of a large chronic subdural hematoma: a case report and review of the literature. Ulus Travma Acil Cerrahi Derg. 2009; 15(1):95-98.

15. Lee CH, Kang DH, Hwang SH, Park IS, Jung JM, Han $J W$. Spontaneous rapid reduction of a large acute subdural hematoma. J Korean Med Sci. 2009; 24(6):1224-1226.

16. Ram Z, Hadani M, Sahar A, Spiegelmann R. Continuous irrigation-drainage of the subdural space for the treatment of chronic subdural hematoma: a prospective clinical trial. Acta Neurochir (Wein). 1993; 120(1-2):40-43.

17. Aoki N. Subdural tapping and irrigation for treatment of chronic subdural hematoma in adults. Neurosurgery. 1984; 14(5):545-548.

18. Asfora WT, Schwebach L. A modified technique to treat chronic and sub acute subdural hematoma: technical note. Surg Neurol. 2003; 59(4):329-332.

19. Gurelik M, Aslan A, Gurelik B, Ozum U, Karadag O, Kars HZ. Asafe and effective method for treatment of chronic subdural hematoma. Can J Neurol Sci. 2007; 34(1):84-87.

20. Zakaraia AM, Adnan JS, Haspani MS, Naing NN, Abdullah JM. Outcome of 2 different types of operative techniques practiced for chronic subdural hematoma in Malaysia: an analysis. Surg Neurol. 2008; 69(6):608-615.

21. Abouzari M, Rashidi A, Rezaii J, et al. The role of postoperative patient posture in the recurrence of traumatic chronic subdural hematoma after burr hole surgery. Neurosurgery. 2007; 61(4):794-797.

22. Nakajima H, Yasui T, Nishikawa M, Kishi H, Kan M. The role of postoperative patient posture in the recurrence of chronic subdural hematoma: a prospective randomized trial. Surg Neurol. 2002; 58(6):385-387. 